\title{
The Contrecoup Injury in a Cat Case of Traffic Accident: MRI Findings
}

\author{
Kazutaka YAMADA, Kazuro MIYAHARA, Motoyoshi SATO, Takayoshi MIYABAYASHI ${ }^{1)}$ and Tsuneo HIROSE \\ Department of Veterinary Clinical Radiology, Obihiro University of Agriculture and Veterinary Medicine, Obihiro 080-8555, Japan and \\ ${ }^{1)}$ Department of Small Animal Clinical Sciences, College of Veterinary Medicine, University of Florida, Florida, U.S.A. \\ (Received 5 November 1997/Accepted 19 January 1998)
}

ABSTRACT. The clinical application of MRI of a cat case of traffic accident was examined. On admission, the animal was unconscious and remained so for 2 days. Radiographs disclosed a fracture in the parietal bone. From the temporary unconscious status and the fracture, cerebral damage was suspected and an MRI examination was performed. The contrecoup injury in the cat case of traffic accident which could not be diagnosed by radiography was diagnosed by MRI examination.-KEY wORDs: contrecoup injury, MRI, subdural hematoma.

J. Vet. Med. Sci. 60(5): 647-649, 1998

$\mathrm{X}$-ray diagnosis is generally used to evaluate damages in the chest-abdominal region and fractures in traffic accident cases. However it is difficult to diagnose intracranial tissue damage by radiography. The utility of MRI is widely recognized in the veterinary field, yet there has been no report documenting diagnoses of intracranial tissue damage in traffic accident cases using MRI. This is owing largely to the fact that it is difficult to perform MRI immediately after occurrence of a traffic accident and that it is often the case that the chance for MRI examination is missed because of the rapid clinical progress in most traffic accident cases. Another reason would be that the risk in injured animals may increase due to anesthesia done for preparation for this procedure. We report our experience in MRI diagnosis in a cat case of traffic accident.

Prior to the clinical application of MRI, the optimum scanning parameter was explored in a healthy male cat according to the previously described method [1]. The optimum scanning parameter for the cat head is set at TR/ $\mathrm{TE}=500 / 25 \mathrm{msec}$ in $\mathrm{T}_{1}$ weighted image and $\mathrm{TR} / \mathrm{TE}=1,600 /$ $90 \mathrm{msec}$ in $\mathrm{T}_{2}$ weighted image. Then the clinical case was scanned using these optimum scanning values. The male cat traffic accident case (B.W. $3.5 \mathrm{~kg}$ ) brought in the Veterinary Teaching Hospital of Obihiro University of Agriculture and Veterinary Medicine. On admission, the animal was unconscious and remained so for 2 days. Physical examination revealed traumas in the right head and right forefoot, and radiographs disclosed a fracture in the parietal bone (Fig. 1, arrow). From the temporary unconscious status and the fracture, cerebral damage was suspected and MRI examination was performed on the 12th hospital day. MRI images were obtained with a 0.2 tesla magnetic field strength permanent magnet unit (MRP20EX, Hitachi Medical Corporation, Chiba, Japan) equipped with a quadrature radio frequency coil which resonated at 8.56 $\mathrm{MHz}$. The animal was anesthetized intravenously with pentobarbital sodium at a dose of $25 \mathrm{mg} / \mathrm{kg}$, and placed on spontaneous respiration via endotracheal intubation. MRI scans were then performed. Confirming the animal's recovery from anesthesia, and showing that normal body temperature had been attained, the MRI examination was finished. Intravenous anesthesia was used in this study because inhalation anesthesia apparatus could not be brought in the magnetic field. The retention frame device made of hard urethane which was designed by Nakagawa et al. for small animals to prevent from moving was used [2]. In addition, meglumine gadopentatete (Magnevist ${ }^{\circledR}$, Schering, Osaka, Japan) was administered intravenously at a dose of $0.1 \mathrm{mmol} / \mathrm{kg}$ for detail investigation.

Transverse $T_{1}$ weighted image of the head shows a low signal intensity area in the right parietal lobe where the fracture exists (Fig. 2, a). This area shows a high signal intensity in $\mathrm{T}_{2}$ weighted image (Fig. 2, a) and a contrastenhanced image (Fig. 2, a). In the other section visualizing the other side of the cerebrum, a high signal intensity area along the left frontal margin was observed in $\mathrm{T}_{1}$ weighted image (Fig. 2, b). This area also showed a high signal intensity in $\mathrm{T}_{2}$ weighted image (Fig. 2, b). The high signal intensity areas in the $T_{1}$ and $T_{2}$ weighted images reflected methemoglobin due to subdural hematoma [3]. And the high signal area was observed in the left temporal lobe in $\mathrm{T}_{2}$ weighted image (Fig. 2, c). And contrast-enhanced image (Fig. 2, c) depicted the high signal intensity area correlating to the high signal intensity area observed in the $T_{2}$ weighted image. The enhanced area was due to disruption of the

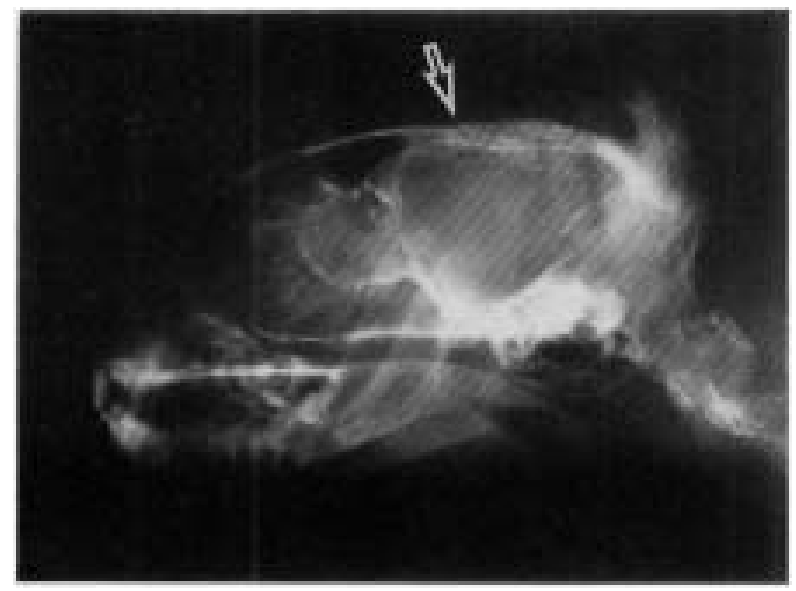

Fig. 1. Radiograph of the head of the cat traffic accident case. Radiograph disclosed a fracture in the parietal bone (arrow). 


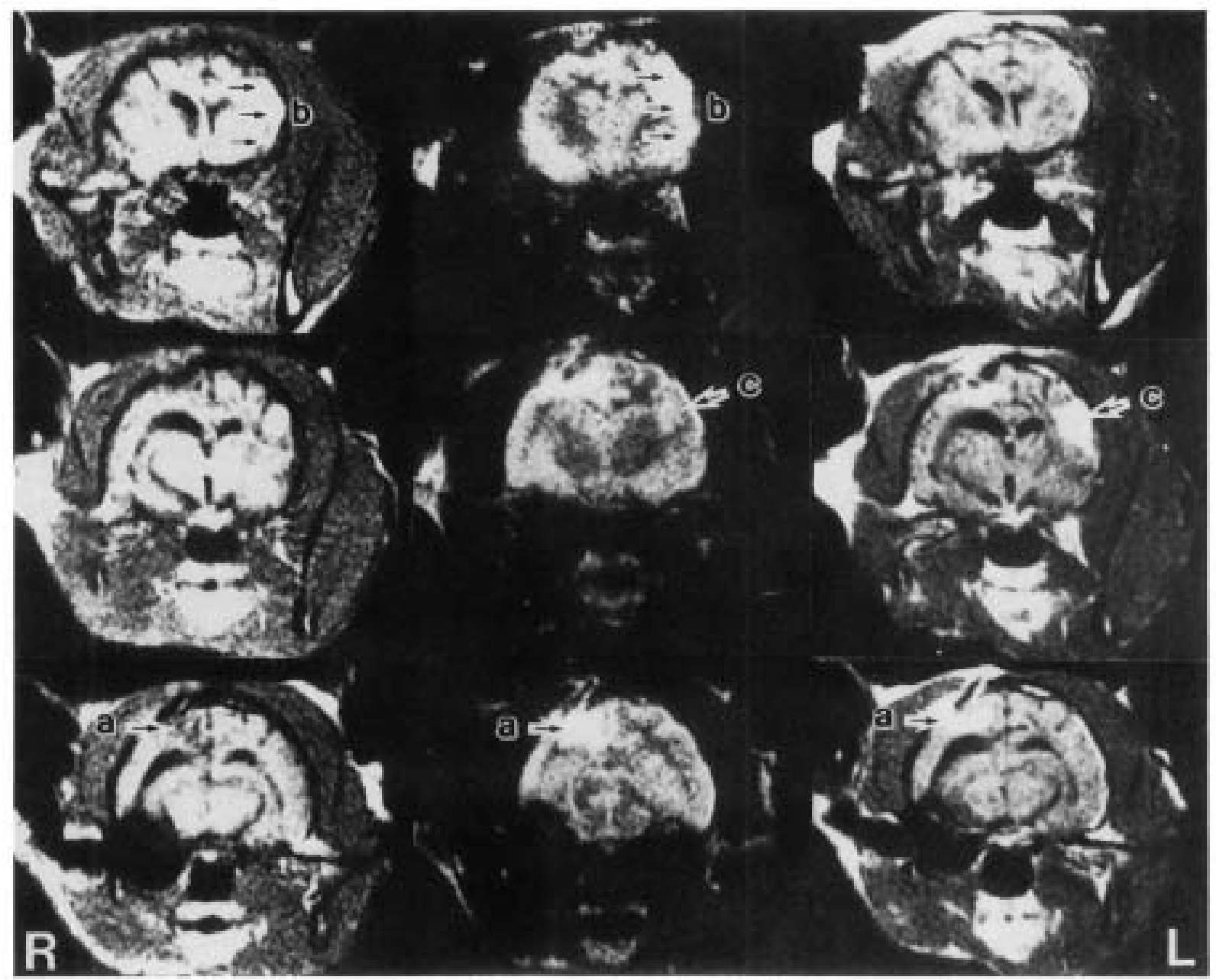

Fig. 2. Transverse images of the head of the cat traffic accident case. (left: $\mathrm{T}_{1}$ weighted image; center: $\mathrm{T}_{2}$ weighted image; right: contrast-enhanced image) A low signal intensity area (a) of the right parietal lobe where the fracture exists was observed in $\mathrm{T}_{1}$ weighted image. This area (a) shows a high signal intensity in $T_{2}$ weighted image and a contrast-enhanced image. A high signal intensity (b) area along the left frontal margin was observed in $\mathrm{T}_{1}$ weighted image. This area (b) also shows a high signal intensity in $\mathrm{T}_{2}$ weighted image. And a high signal area (c) was observed in the left temporal lobe in $\mathrm{T}_{2}$ weighted image. A contrast-enhanced image depicted the high signal intensity area (c) correlating to the high signal intensity area observed in $\mathrm{T}_{2}$ weighted image.

blood-brain barrier by the trauma, indicating that gadolinium was transported from the blood vessel to the cerebral tissue $[4,5]$. These lesions on the opposite symmetric side of the injury represented a contrecoup injury, i.e. a trauma on the side opposite that of the primary where the impact had been received. When the moving head stops suddenly as impacted by some object, the head stops but intracranial brain does not stop, then the brain collides against the other side of the cranium, thus damaging the intracranial brain. The animal died 2 days after the MRI examination. Postmortem examination revealed the trauma (Fig. 3, a) in the right parietal lobe and wide subdural hematoma (Fig. 3, b) in the left parietal and temporal lobes. There was a contrecoup injury in the left temporal lobe (Fig. 3, c). The information obtained from MRI was highly consistent with the necropsy findings. The contrecoup injury in the cat case of traffic accident which could not be diagnosed by radiography were diagnosed by MRI examination.

\section{REFERECES}

1. Yamada, K., Miyahara, K., Sato, M., Hirose, T., Yasugi, Y., Matsuda, Y. and Furuhama, K. 1995. Vet. Radiol. Ultrasound. 36: 523-527

2. Nakagawa, M., Miyahara, K. and Hirose, T. 1993. J. Jpn. Vet. Med. Assoc. 46: 147-150.

3. Hayman, L. A., McArdle, C. B., Taber. K. H., Saleem, A., Baskin, D., Lee, H. S., Kirkpatrick, J. B., Herrick, R. C. and Bryan, R. N. 1989. Am. J. Neuro. Radiol. 10: 681-686.

4. Yamada, K., Jinbo, T., Miyahara, K., Sato, M., Hirose, T., Tateno, Y., Ikehira, H., Sugihara, H. and Furuhama, K. 1996. J. Vet. Med. Sci. 58: 389-396.

5. Yamada, K., Miyahara, K., Sato, H., Nakayama, W., Sato, M., Hirose, T., Tateno, Y., Ikehira, H., Sugihara, H. and Furuhama, K. 1996. J. Vet. Med. Sci. 58: 291-295. 


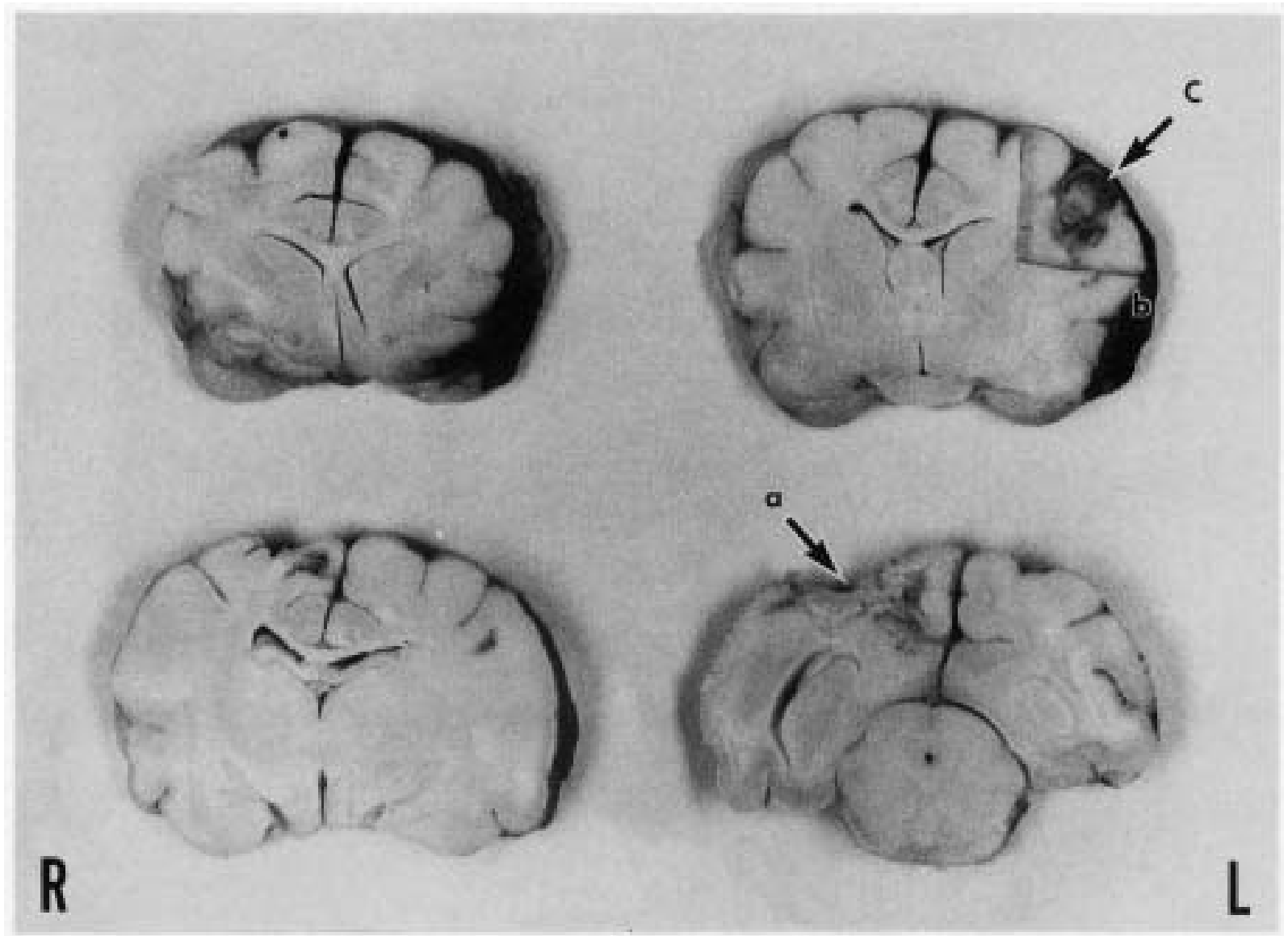

Fig. 3. Transverse section of the brain of the cat traffic accident case.

a: The trauma in the right parietal lobe, b: The subdural hematoma in the left parietal and temporal lobe, c: The contrecoup injury in the left temporal lobe. 